\title{
ON THE SPECTRA OF SCHRÖDINGER AND JACOBI \\ OPERATORS WITH COMPLEX-VALUED QUASI-PERIODIC \\ ALGEBRO-GEOMETRIC COEFFICIENTS
}

Vladimir Batchenko

Dr. Fritz Gesztesy, Dissertation Supervisor

\begin{abstract}
In this thesis we characterize the spectrum of one-dimensional Schrödinger operators $H=-d^{2} / d x^{2}+V$ in $L^{2}(\mathbb{R} ; d x)$ with quasi-periodic complex-valued algebrogeometric potentials $V$ (i.e., potentials $V$ which satisfy one (and hence infinitely many) equation(s) of the stationary Korteweg-de Vries (KdV) hierarchy) associated with nonsingular hyperelliptic curves. The spectrum of $H$ coincides with the conditional stability set of $H$ and can explicitly be described in terms of the mean value of the inverse of the diagonal Green's function of $H$.

As a result, the spectrum of $H$ consists of finitely many simple analytic arcs and one semi-infinite simple analytic arc in the complex plane. Crossings as well as confluences of spectral arcs are possible and discussed as well. These results extend to the $L^{p}(\mathbb{R} ; d x)$-setting for $p \in[1, \infty)$.

In addition, we apply these techniques to the discrete case and characterize the spectrum of one-dimensional Jacobi operators $H=a S^{+}+a^{-} S^{-}-b$ in $\ell^{2}(\mathbb{Z})$ assuming $a, b$ are complex-valued quasi-periodic algebro-geometric coefficients. In analogy to the case of Schrödinger operators, we prove that the spectrum of $H$ coincides with the conditional stability set of $H$ and can also explicitly be described in terms of the mean value of the Green's function of $H$. The qualitative behavior of the spectrum of $H$ in the complex plane is similar to the Schrödinger case: the spectrum consists of finitely many bounded simple analytic arcs in the complex plane which may exhibit crossings as well as confluences.
\end{abstract}

